\title{
STUDIES ON COMBINING ABILITY FOR YIELD AND ITS COMPONENTS IN RICE USING LINE X TESTER MATING DESIGN UNDER SALINE SOIL CONDITIONS \\ Sultan, M. S. ; M. A. Abdel-Moneam ${ }^{1}$; H. M. Hassan ${ }^{2}$ and E. M. Daher ${ }^{2}$ \\ ${ }^{1}$ Agronomy Department, Fac. of Agric. Mansoura University, Egypt. ${ }^{2}$ Rice Research Section, Field Crops Res. Inst., ARC, Giza, Egypt.
}

\begin{abstract}
A Study on combining ability and genetic parameters were conducted on 39 F1 hybrids along with sixteen rice genotypes (thirteen lines and three testers) to understand the pattern of inheritance of yield and its components for selecting superior genotypes. The experiment was carried out using line $x$ tester mating design, during 2011 and 2012 rice growing seasons at the Lysimeter and the Experimental Farm of Rice Research and Training Center, Sakha, Kafr El-Sheikh, Egypt. Both GCA and SCA variances were found to be significant or highly significant for all studied traits. Sakha 102 and Giza 177 rice genotypes were the best general combiners due to highly significant GCA effects for number of days to $50 \%$ heading under normal conditions. Moreover, Sakha 106 was the best general combiner for number of days to $50 \%$ heading at two levels $4000 \mathrm{ppm}$ (S1) and $6000 \mathrm{ppm}$ (S2) of salinity. The genotype, Gaori was the best general combiner for plant height under all conditions and for number of filled grains/panicle and sterility\% under normal conditions. IEET1444 was the best general combiner for number of panicles/plant, salinity index and grain yield/plant under two levels (S1 and S2) of salinity. While, Wab 880SG-33 was the best general combiners for 1000-grain weight under all conditions, and for number of filled grains/panicle under two levels(S1 and S2) of salinity. Suweon 345 was the best general combiner for number of panicles/plant under normal conditions and for sterility\% under two levels (S1 and S2) of salinity. On the other hand, the crosses, IET 1444 x Sakha 104, Wab 880SG-33 x Sakha104, BG 34-8 x Giza 178 and Gz 1368-S-5-4 x Giza 178 were found to be the best cross combinations for most of the studied traits, these crosses had high and significant SCA in desirable direction under normal and two levels of salinity condition. The highest estimated values of phenotypic (Vph) and genotypic $(\mathrm{Vg})$ variances were observed for number of filled grains/panicle and sterility \% indicating better scope for the genetic improvement in these two mentioned traits. High broad-sense heritability, coupled with high genetic advance, were observed for number of filled grains/panicle, sterility \% and plant height under normal and two levels of salinity condition, indicating the role of additive genetic variance in the expression of these traits and direct selection might be highly effective in early generations to improve these traits under normal and two levels of salinity. Highly significant positive correlation coefficients were recorded between grain yield per plant and each of number of panicles/plant, number of filled grains/panicle and 1000- grain weight under normal and two levels of salinity conditions.

Keywords: Rice, combining ability, salinity, genetic advance, yield and line $x$ tester design
\end{abstract}


Sultan, M. S. et al.

\section{INTRODUCTION}

Rice is one of the most important cereal crops, which is a very popular staple food in many developing countries such as Egypt. Grain yield is one of the most breeding objectives. Considerable efforts have been made by breeders to study the genetic expression for numerous characters contributing to yield potential including days to $50 \%$ heading, plant height, number of panicles/ plant, 1000-grain weight, number of filled grains/ panicle, salinity index, sterility \% and grain yield traits.

Rice is considered a salt-sensitive crop species it has a salinity threshold of $3 \mathrm{dS} / \mathrm{m}$, with a $12 \%$ reduction in yield per $\mathrm{dSm}^{-1}$, beyond this threshold. Therefore, rice yields can be reduced by up to $50 \%$ when grown under moderate $\left(6 \mathrm{dSm}^{-1}\right)$ salinity conditions (Zeng et al., 2002). In general, rice plants are very sensitive to salinity stress at young seedling stages, relatively tolerant at the later vegetative stages, and sensitive again during reproduction (Flowers and Yeo,. 1981 and Lutts et al., 1995).

The decreasing of rice productivity in salt-affected areas can be addressed through an integrated approach involving both reclamation and management strategies, as well as enhanced genetic tolerance. However, management practices are not always feasible in the long run, as in coastal areas where salt stress is seasonal or in inlands when reclamation costs are prohibitive. In both cases, developing salt-tolerant varieties seems more feasible to enhance the productivity of these marginal lands. Rice is recommended as a crop best suited for salt affected soils because it can grow well under flooded conditions that can help in leaching harmful salts (Ismail et al., 2008)

The success of a plant breeding program greatly depends on right choice of parents for hybridization and the gene action of different economic traits. Combining ability analysis provides such information so as to frame the breeding program effectively. The line $\mathrm{x}$ tester analysis gives reliable information about the nature and magnitude of gene action and combining ability effects present in the genetic materials. Dhillon. (1975) pointed out that the combining ability gives useful information on the choice of parents in terms of expected performance of the hybrids and their progenies. The line $x$ tester analysis method is used to breed both self and cross-pollinated plants and to estimate favorable parents and crosses, and their general and specific combining abilities (Kempthorne, 1957).

The performance of parent may not necessarily reveal it to be a good or poor combiner. Therefore, gathering information on nature of gene effects and their expression in terms of combining ability is necessary. At the same time, it also elucidates the nature of gene action involved in the inheritance of characters. General combining ability (GCA) is attributed to additive gene effects and additive $x$ additive epistasis and is theoretically fixable. On the other hand, specific combining ability attributable to non-additive gene action may be due to dominance or epistasis or both and is non-fixable. The presence of non-additive genetic variance is the primary justification for initiating the hybrid program (Pradhan et al., 2006). The main objectives of 
the present investigation are to: 1 - Study the general and specific combining abilities and genetic parameters for yield and its components traits under normal and saline conditions. 2- Study the phenotypic correlation coefficients among all possible pairs of the studied characters under normal and saline conditions.

\section{MATERIALS AND METHODS}

The experiment was carried out using line $\mathrm{x}$ tester mating design, during 2011 and 2012 rice growing seasons at the Lysimeter and the Experimental Farm of Rice Research and Training Center (RRTC), Sakha, Kafr El-Sheikh, Egypt. The experimental material of the present study comprised thirteen lines, namely, IEET1444, Gz 1368-S-5-4, BG 34-8, Agami M1, Giza 182 and Gaori (tolerant genotypes for salinity), Wab 880SG-33, Suweon 345 and Yabani lulu (moderately tolerant to salinity), and Wab 880p1, Giza 177, Sakha 106 and Sakha 105 (sensitive genotypes to salinity); and three testers namely, Sakha 104 and Giza 178(tolerant genotypes to salinity) and Sakha 102 (sensitive genotype to salinity) with diverse pure and elite genotypes of rice. The parents were selected on the basis of different genetic and geographical origin. The testers used for this study were selected according to their subcontinent origin and adaption to Egyptian locality and their yield potential was up to the mark (The lines were originated from RRTC, Egypt). In 2011, the thirteen lines and three testers were grown at RRTC farm in three successive dates of planting with ten days intervals in order to overcome the differences in flowering time between parents. At flowering time, hybridization between the parents was done, 30 days old seedlings of each parent were individually transplanted in the permanent field in seven rows following the technique proposed by Jodon (1938) to produce their $F 1$ using line $x$ tester mating design.

In 2012 season, seeds of the line $x$ tester $F 1$ Hybrids and their parents were sown in dry seedbed. After thirty days from sowing, seedlings of each F1 hybrids and their parents were transplanted under normal and saline soil conditions in Lysimeter at randomized complete block design experiment with three replications. Each replicate comprised 5 row for each $F_{1}$ cross and $20 x$ $20 \mathrm{~cm}$ apart was maintained between rows and seedlings. All agricultural practices such as sowing date, fertilizer application and weed control were applied as recommended. Irrigation and drainage cycle were accurately controlled in normal and saline conditions. The saline soil conditions were adjusted to $4000 \mathrm{ppm}$ (S1) and $6000 \mathrm{ppm}$ (S2), in addition, the normal soil conditions were irrigated by pumping water. The mean value of electrical conductivity $(E C)$ of irrigation water is $0.77 \mathrm{mmhos} / \mathrm{cm}$ for normal, otherwise, 6.9 and $10.6 \mathrm{mmhos} / \mathrm{cm}$ at $25^{\circ} \mathrm{C}$ for the two levels of saline soil condition, respectively. The water was artificially salinized by applying sodium chloride $(\mathrm{NaCL})$ and calcium chloride (CaCL2). The Lysimeter plot is concrete beds ( $1 \mathrm{~m}$ width $\times 2 \mathrm{~m}$ length) filled with soil to $100 \mathrm{~cm}$ depth in three layers, i.e., 60 $\mathrm{cm}$ clay at surface layer, $20 \mathrm{~cm}$ sand at the middle layer and $20 \mathrm{~cm}$ gravel at bottom layer. Data were collected for vegetative, yield and its components 
traits; number of days to $50 \%$ heading, plant height $(\mathrm{cm})$, number of panicles/plant, number of filled grains/ panicle, sterility \%, 1000- grain weight (g), salinity tolerance index and grain yield /plant (g) according to the standard evaluation system of IRRI (1996).

Combining ability analysis was done using line $x$ tester. The variances for general combining ability and specific combining ability were tested against their respective error variances derived from ANOVA reduced to mean level. Significance test for GCA and SCA effects were performed using T-test. The following variance components were estimated based on the expectations of mean squares according to Kempthorne (1957).

\section{RESULTS AND DISCUSSION}

\section{Estimates of general and specific combining ability effects General combining ability effects:}

The estimates of general combining ability effects consider an important indicator of the potential of parental lines for generating superior breeding populations. A negligible or negative combining ability effect indicates a poor ability to transfer its genetic superiority to hybrids. The largest significant positive values have the largest effects. On the other hand, the largest significant negative values have the smallest effects, except in case of sterility \%, duration (days) and plant height traits. Obviously, under normal conditions, Table 1 indicated that the line Giza 177 and the tester Sakha 102 were the best general combiners for earliness, plant height and 1000 - grain weight. While, Gaori was the best general combiners for plant height, number of filled grains per panicle and sterility\%. In addition, Suweon 345 rice line was a good general combiner for increasing number of panicles/plant, 1000- grain weight, number of filled grains/panicle, sterility\% and grain yield/plant. While, Wab 880SG-33 and Wab 880- 11 were the best general combiners for 1000- grain weight. The results revealed that among the studied parents, Gz 1368-S-5-4 followed by Suweon 345 and Giza 178 were the best general combiners for grain yield/plant. However, some parents with high mean values exhibited low GCA effects. Hence, both performances per se and GCA effects should be taken into account for parental selection.

It is obvious that none of the parents were found to be good combiners for all the studied traits. Hence, it would be desirable to have multiple crosses involving the parents, viz., IEET 1444, Giza 178, Wab 880SG-33, Suweon 345, Gaori and Gz 1368-S-5-4 for practicing a selection in the segregating generations to isolate superior genotypes under normal conditions.

On the other hand, under level $\mathrm{S} 1$ of salinity condition, results in the Table 1 show that lines, Sakha106, Wab 880SG-33, Wab 880-p1 and IEET1444 and tester, Sakha 102 were the best general combiners for earliness. While, Gaori, Yabani lulu, Giza 177and Wab 880-p1 were the best general combiners for plant height. In addition, the genotype IEET 1444 was good general combiners for improving number of panicles per plant, salinity 
tolerance index and grain yield/plant. While, Wab 880SG-33 was the best general combiner for 1000- grain weight and number of filled grains per panicle. The results revealed that among the studied parents, Suweon 345 followed by Wab 880SG-33 and Yabani lulu were the best general combiners for sterility\%. Moreover, IEET1444 and Gz1368-S-5-4 were the best general combiners for grain yield/plant. Regarding the level (S2) of salinity condition, results in Table (1) exhibited that Sakha 106 and IEET1444 were the best general combiners for earliness.

Table 1: Estimates of general combining ability effects for yield and its related traits for parental genotypes.

\begin{tabular}{|c|c|c|c|c|c|c|}
\hline \multirow{2}{*}{ Genotypes } & \multicolumn{3}{|c|}{ Days to $50 \%$ heading } & \multicolumn{3}{|c|}{ Plant height $(\mathrm{cm})$} \\
\hline & $\mathbf{N}$ & S1 & S2 & $\mathbf{N}$ & S1 & S2 \\
\hline$L_{1}-$ Wab 880SG-33 & 1.92 & $-3.92^{* *}$ & -1.05 & $11.08^{* *}$ & $3.89^{* *}$ & $-6.72^{* \star}$ \\
\hline $\mathrm{L}_{2}-$ Wab 880-p1 & -2.08 & $-5.70^{* \star}$ & $-4.05^{* *}$ & $12.74^{* *}$ & $-3.89^{* *}$ & -2.05 \\
\hline L3- IEET 1444 & 0.03 & $-5.92^{\star \star}$ & $-5.39^{* *}$ & $-4.59^{\star *}$ & 0.22 & 2.62 \\
\hline L4- Gaori & -2.63 & 1.08 & 1.28 & $-19.92^{\star *}$ & $-17.11^{* *}$ & $-14.05^{\star \star}$ \\
\hline L5- Giza 177 & $-7.74^{\star \star}$ & $3.30^{*}$ & $-6.05^{\star \star}$ & $-11.26^{\star *}$ & $-6.22^{\star \star}$ & $-5.39^{\star *}$ \\
\hline L6- Giza 182 & $3.59^{* \star}$ & 0.74 & -2.27 & $-3.92^{* *}$ & $-3.11^{*}$ & -1.05 \\
\hline L7- Sakha 106 & $-5.08^{\star \star}$ & $-6.70^{* \star}$ & $-10.39^{* \star}$ & $-13.92^{\star *}$ & 2.56 & 2.62 \\
\hline L_- Sakha 105 & $-4.41^{* *}$ & $5.30^{* *}$ & -0.72 & $-14.81^{* *}$ & -2.11 & -1.39 \\
\hline Lg- Suweon 345 & 1.26 & 1.74 & 2.62 & $9.19^{* *}$ & -2.11 & $-3.39^{*}$ \\
\hline$L_{10}-$ Gz 1368-S-5-4 & -0.08 & $-2.81^{*}$ & $2.95^{\star}$ & $11.41^{\star *}$ & $8.89^{* *}$ & $9.95^{\star \star}$ \\
\hline$L_{11}-B G \quad 34-8$ & $6.92^{\star \star}$ & $5.08^{\star \star}$ & $8.39^{\star \star}$ & $19.74^{\star *}$ & $19.22^{\star \star}$ & $18.28^{* *}$ \\
\hline $\mathrm{L}_{12}$ - Agami M1 & $5.26^{\star *}$ & $3.74^{* \star}$ & $6.39^{* \star}$ & $6.86^{* *}$ & $9.89^{* *}$ & $11.28^{* *}$ \\
\hline$L_{13}$ - Yabani lulu & $3.03^{*}$ & $4.08^{\star \star}$ & $8.28^{\star *}$ & -2.59 & $-10.11^{\star *}$ & $-10.72^{\star \star}$ \\
\hline S.E $\left(g_{i}\right)$ & 1.33 & 1.40 & 1.41 & 1.32 & 1.35 & 1.37 \\
\hline S.E ( $\left.g_{1}-g_{j}\right)$ & 1.88 & 1.97 & 1.99 & 1.87 & 1.90 & 1.93 \\
\hline L.S.D 0.05 & 2.66 & 2.8 & 2.82 & 2.64 & 2.7 & 2.74 \\
\hline 0.01 & 3.54 & 3.72 & 3.75 & 3.51 & 3.59 & 3.64 \\
\hline T1- Sakha 102 & $-8.67^{\star \star}$ & $-6.03^{*}$ & -4.77 & $-5.64^{*}$ & -1.80 & -0.59 \\
\hline$T_{2}$ - Sakha 104 & $9.85^{\star \star}$ & 5.21 & $6.54^{*}$ & 4.59 & -4.93 & $-6.13^{*}$ \\
\hline$T_{3}$ - Giza 178 & -1.18 & 0.82 & -1.77 & 1.05 & $6.74^{*}$ & $6.72^{*}$ \\
\hline S.E (gi $)$ & 2.76 & 2.91 & 2.93 & 2.75 & 2.80 & 2.85 \\
\hline S.E (gt-gij) & 0.90 & 0.95 & 0.96 & 0.90 & 0.91 & 0.93 \\
\hline LS D at 0.05 & 5.52 & 5.82 & 5.86 & 5.5 & 5.6 & 5.7 \\
\hline at 0.01 & 7.34 & 7.74 & 7.79 & 7.32 & 7.45 & 7.58 \\
\hline
\end{tabular}


Sultan, M. S. et al.

Table 1 : Cont.

\begin{tabular}{|c|c|c|c|c|c|c|}
\hline \multirow{2}{*}{ Genotypes } & \multicolumn{3}{|c|}{ No. of panicles/plant } & \multicolumn{3}{|c|}{ 1000-grain weight (g) } \\
\hline & $\mathbf{N}$ & S1 & S2 & $\mathbf{N}$ & $\mathrm{S} 1$ & S2 \\
\hline$L_{1}-$ Wab 880SG-33 & $-8.21^{\star *}$ & -2.46 & -1.00 & $0.40^{* *}$ & $0.36^{*}$ & $0.38^{*}$ \\
\hline $\mathrm{L}_{2}-\mathrm{Wab}$ 880-p1 & -0.55 & -0.46 & 1.00 & $0.22^{* \star}$ & 0.09 & 0.12 \\
\hline$L_{3}$ - IEET 1444 & $5.45^{\star \star}$ & $4.54^{\star \star}$ & $5.33^{* \star}$ & $-0.21^{\star \star}$ & 0.01 & -0.19 \\
\hline $\mathrm{L}_{4}-\mathrm{Gaori}$ & $-3.88^{*}$ & -0.46 & -2.33 & $-0.17^{\star \star}$ & -0.11 & 0.09 \\
\hline$L_{5}-$ Giza 177 & -2.55 & $-3.46^{*}$ & $-3.00^{*}$ & $0.13^{\star \star}$ & 0.20 & 0.03 \\
\hline$L_{6}-$ Giza 182 & -2.44 & 1.87 & 0.67 & 0.04 & 0.07 & 0.22 \\
\hline$L_{7-}$ Sakha 106 & $-3.55^{* *}$ & -2.46 & $-3.33^{*}$ & $0.15^{\star \star}$ & 0.13 & 0.02 \\
\hline$L_{8}$ - Sakha 105 & 1.45 & -1.80 & -1.00 & -0.01 & 0.06 & 0.06 \\
\hline$L_{9}-$ Suweon 345 & $10.45^{\star \star}$ & 0.54 & 1.33 & $0.17^{\star \star}$ & 0.06 & 0.07 \\
\hline$L_{10}-$ Gz 1368-S-5-4 & $4.45^{\star \star}$ & $4.21^{\star \star}$ & $4.33^{* *}$ & -0.04 & -0.04 & -0.02 \\
\hline$L_{11}-B G 34-8$ & 1.12 & $3.87^{* \star}$ & 2.00 & $-0.41^{\star \star}$ & $-0.50^{* \star}$ & $-0.40^{*}$ \\
\hline$L_{12}$ - Agami M1 & -1.21 & -0.13 & 0.00 & $-0.24^{\star *}$ & -0.12 & 0.11 \\
\hline \begin{tabular}{|l|l} 
& $L_{13^{-}}$Yabani lulu \\
\end{tabular} & -0.55 & $-3.80^{\star \star}$ & $-4.00^{\star \star}$ & -0.04 & -0.19 & $-0.48^{\star \star}$ \\
\hline S.E $\left(g_{i}\right)$ & 1.28 & 1.32 & 130 & 0.03 & 0.16 & 0.17 \\
\hline S.E $\left(g_{1}-g_{j}\right)$ & 1.82 & 1.87 & 1.84 & 0.04 & 0.22 & 0.25 \\
\hline L.S.D 0.05 & 2.56 & 2.64 & 2.60 & 0.06 & 0.32 & 0.34 \\
\hline \begin{tabular}{|l|}
0.01 \\
\end{tabular} & 3.40 & 3.51 & 3.5 & 0.08 & 0.43 & 0.45 \\
\hline$T_{1}$ - Sakha 102 & -3.73 & -2.03 & -0.74 & $0.15^{*}$ & 0.41 & 0.10 \\
\hline$T_{2}$ - Sakha 104 & -2.01 & -4.41 & -2.97 & 0.07 & 0.27 & 0.13 \\
\hline$T_{3-\text { Giza } 178}$ & $5.74^{*}$ & $6.44^{*}$ & 3.72 & $-0.22^{\star *}$ & $-0.69^{*}$ & -0.23 \\
\hline S.E $\left(g_{i}\right)$ & 2.67 & 2.75 & 2.71 & 0.07 & 0.33 & 0.36 \\
\hline S.E $\left(g t-g_{j}\right)$ & 0.87 & 0.90 & 0.88 & 0.02 & 0.11 & 0.12 \\
\hline LS D at 0.05 & 5.34 & 5.5 & 5.4 & 0.14 & 0.66 & 0.72 \\
\hline \begin{tabular}{|l|} 
at 0.01 \\
\end{tabular} & 7.10 & 7.32 & 7.2 & 0.19 & 0.88 & 0.96 \\
\hline
\end{tabular}

*and ${ }^{\star *}$ significant at 0.05 and 0.01 probability levels, respectively.

Abbreviations: N, normal conditions, S1, first level of salinity (4000ppm), S2, second level of salinity $(6000 \mathrm{ppm})$

Table 1 : Cont.

\begin{tabular}{|c|c|c|c|c|c|}
\hline \multirow{2}{*}{$\begin{array}{c}\text { No. } \\
\text { Genotype }\end{array}$} & \multicolumn{3}{|c|}{ No. of filled grains/panicle } & \multicolumn{2}{|c|}{ salinity index } \\
\hline & $\mathbf{N}$ & S1 & S2- & S1 & S2 \\
\hline$L_{1}-$ Wab 880SG-33 & $-9.46^{\star \star}$ & $26.34^{\star \star}$ & $16.92^{\star \star}$ & -1.37 & -1.58 \\
\hline $\mathrm{L}_{2}-$ Wab 880-p1 & $24.54^{\star \star}$ & $-3.21^{\star}$ & $-9.20^{\star \star}$ & $-6.30^{\star \star}$ & $-2.63^{*}$ \\
\hline $\mathrm{L}_{3}$ - IEET 1444 & $7.21^{\star \star}$ & $10.79^{* \star}$ & $3.58^{*}$ & $15.96^{\star \star}$ & $7.99^{\star \star}$ \\
\hline $\mathrm{L}_{4}-\mathrm{Gaori}$ & $44.54^{\star \star}$ & $-13.99^{\star \star}$ & $-14.42^{\star \star}$ & $-7.22^{\star \star}$ & -1.17 \\
\hline L- Giza 177 & $-26.13^{\star \star}$ & $5.23^{\star \star}$ & 1.14 & $-3.92^{\star \star}$ & 0.38 \\
\hline $\mathrm{L}_{6^{-}}$Giza 182 & -0.13 & -1.10 & $5.14^{\star \star}$ & -0.25 & -2.12 \\
\hline$L_{7}$ - Sakha 106 & $-28.13^{\star \star}$ & $17.12^{\star \star}$ & -1.64 & -0.09 & $2.22^{*}$ \\
\hline$L_{8}$ - Sakha 105 & $6.87^{* *}$ & 1.79 & $10.03^{* *}$ & $-4.77^{* *}$ & -0.33 \\
\hline$L_{9}-$ Suweon 345 & $28.54^{* *}$ & $13.12^{\star \star}$ & $12.36^{\star \star}$ & $-10.32^{\star \star}$ & $-5.79^{\star \star \star}$ \\
\hline $\mathrm{L}_{10}-\mathrm{Gz} 1368-\mathrm{S}-5-4$ & $7.21^{\star *}$ & -1.88 & $11.69^{* *}$ & $-8.11^{\star \star}$ & $-5.09^{* \star}$ \\
\hline $\mathrm{L}_{11}-\mathrm{BG} 34-8$ & $-73.80^{\star \star}$ & $-31.33^{\star \star}$ & $-11.20^{* *}$ & $11.43^{\star \star}$ & $6.32^{* *}$ \\
\hline $\mathrm{L}_{12}$ - Agami M1 & $6.54^{* \star}$ & $-3.77^{\star}$ & $-3.53^{\star}$ & $6.19^{\star \star}$ & $-2.87^{\star}$ \\
\hline $\mathrm{L}_{13}$ - Yabani lulu & $12.21^{\star \star}$ & $-19.10^{\star \star}$ & $-20.86^{\star \star}$ & $8.76^{\star \star}$ & $4.66^{\star \star}$ \\
\hline S.E $\left(g_{i}\right)$ & 1.45 & 1.53 & 1.56 & 1.04 & 1.08 \\
\hline S.E $\left(g_{1}-g_{j}\right)$ & 2.05 & 2.17 & 2.20 & 1.48 & 1.52 \\
\hline $\begin{array}{ll}\text { L.S.D } & 0.05\end{array}$ & 2.9 & 3.06 & 3.12 & 2.08 & 2.16 \\
\hline 0.01 & 3.86 & 4.07 & 4.15 & 2.77 & 2.87 \\
\hline$T_{1}$ - Sakha 102 & $-13.72^{* *}$ & $-11.38^{\star *}$ & $-6.86^{\star}$ & $4.75^{*}$ & $5.71^{*}$ \\
\hline$T_{2}-$ Sakha 104 & 2.28 & -2.12 & -5.48 & -3.76 & -4.06 \\
\hline$T_{3}-$ Giza 178 & $11.44^{\star \star}$ & $13.50^{\star \star}$ & $12.34^{\star \star}$ & -0.99 & -1.65 \\
\hline S.E $\left(g_{i}\right)$ & 3.02 & 3.19 & 3.24 & 2.17 & 2.24 \\
\hline S.E $\left(g t-g_{i}\right)$ & 0.99 & 1.04 & 1.06 & 0.71 & 0.73 \\
\hline LS D at 0.05 & 6.04 & 6.38 & 6.48 & 4.34 & 4.48 \\
\hline at 0.01 & 8.03 & 8.49 & 8.62 & 5.77 & 5.96 \\
\hline
\end{tabular}

${ }^{*}$ and ${ }^{* *}$ significant at 0.05 and 0.01 probability levels, respectively. Abbreviations: $N$ normal conditions, S1, first level of salinity (4000ppm), S2, second level of salinity (6000ppm) 
Table 1: Cont.

\begin{tabular}{|c|c|c|c|c|c|c|}
\hline \multirow{2}{*}{ Genotype } & \multicolumn{3}{|c|}{ Sterility \% } & \multicolumn{3}{|c|}{ Grain yield/ plant (g) } \\
\hline & $\mathbf{N}$ & S1 & S2 & $\mathbf{N}$ & S1 & S2 \\
\hline$L_{1}-$ Wab 880SG-33 & $-11.29^{\star \star}$ & $-12.19^{\star *}$ & $-15.54^{\star *}$ & 2.79 & 0.71 & -0.07 \\
\hline $\mathrm{L}_{2}-\mathrm{Wab}$ 880-p1 & $-6.51^{\star \star}$ & $-5.63^{\star \star}$ & 2.68 & $-13.47^{\star \star \star}$ & $-6.29^{\star \star}$ & $-3.10^{*}$ \\
\hline$L_{3}-$ IEET 1444 & $6.03^{\star *}$ & 1.17 & $4.54^{\star \star}$ & -0.52 & $12.30^{\star *}$ & $6.26^{\star *}$ \\
\hline$L_{4}$ - Gaori & $-18.28^{\star \star}$ & 1.90 & $11.29^{\star \star}$ & $-6.33^{\star \star}$ & $-5.14^{\star \star}$ & -1.13 \\
\hline $\mathrm{L}_{5}-$ Giza 177 & -2.73 & $-6.69^{\star \star}$ & $-4.71^{\star \star}$ & $-5.79^{\star \star}$ & $-500^{\star \star}$ & -0.16 \\
\hline$L_{6^{-}}$Giza 182 & $-3.46^{*}$ & $6.65^{\star \star}$ & $10.11^{\star \star}$ & $5.53^{\star \star}$ & -0.32 & -1.96 \\
\hline L7- Sakha 106 & $-4.46^{\star *}$ & $-7.21^{\star *}$ & -0.55 & $-10.49^{\star *}$ & -1.80 & -0.16 \\
\hline$L_{8^{-}}$Sakha 105 & $-7.36^{\star *}$ & -0.60 & $-10.28^{* *}$ & $6.43^{\star *}$ & -1.10 & 1.98 \\
\hline$L_{9}$ - Suweon 345 & $-10.53^{\star \star}$ & $-15.31^{\star *}$ & $-20.90^{\star *}$ & $17.37^{\star *}$ & $-4.08^{*}$ & $-3.54^{*}$ \\
\hline $\mathrm{L}_{10^{-}} \mathrm{Gz} 1368-\mathrm{S}-5-4$ & $5.71^{\star \star}$ & $7.89^{\star \star}$ & $3.56^{*}$ & $37.35^{\star \star}$ & $5.53^{\star \star}$ & $3.96^{\star *}$ \\
\hline$L_{11}-B G \quad 34-8$ & $40.04^{\star *}$ & $29.27^{\star \star}$ & $18.59^{\star \star}$ & $-19.32^{\star *}$ & 1.16 & -0.62 \\
\hline$L_{12^{-}}$Agami M1 & $14.24^{\star \star}$ & $8.33^{\star *}$ & 2.59 & -3.01 & $4.68^{\star *}$ & $-3.92^{\star *}$ \\
\hline$L_{13^{-}}$Yabani lulu & -1.41 & $-7.58^{\star \star}$ & -1.39 & $-10.53^{\star \star}$ & 1.35 & 2.47 \\
\hline S.E $\left(g_{i}\right)$ & 1.38 & 1.55 & 1.48 & 1.52 & 1.68 & 1.36 \\
\hline S.E $\left(g_{\mathrm{l}}-g_{\mathrm{j}}\right)$ & 1.95 & 1.20 & 2.10 & 2.15 & 2.37 & 1.93 \\
\hline L.S.D 0.05 & 2.76 & 3.1 & 2.96 & 3.04 & 3.36 & 2.72 \\
\hline 0.01 & 3.67 & 4.12 & 3.94 & 4.04 & 4.47 & 3.62 \\
\hline$T_{1}$ - Sakha 102 & -4.13 & $7.37^{*}$ & $-6.34^{*}$ & $-19.85^{\star \star}$ & $-7.86^{*}$ & $-5.75^{\star}$ \\
\hline $\mathrm{T}_{2^{-}}$Sakha 104 & $6.21^{*}$ & -2.06 & 2.63 & $9.22^{\star *}$ & -1.06 & 1.17 \\
\hline$T_{3^{-}}$Giza 178 & -2.08 & -5.31 & 3.71 & $10.63^{\star *}$ & $8.91^{*}$ & 4.58 \\
\hline S.E $\left(g_{i}\right)$ & 2.87 & 3.24 & 3.09 & 3.17 & 3.49 & 2.84 \\
\hline S.E $\left(g t-g_{j}\right)$ & 0.94 & 1.06 & 1.01 & 1.03 & 1.14 & 0.93 \\
\hline LS D at 0.05 & 5.74 & 6.48 & 6.18 & 6.34 & 6.98 & 5.68 \\
\hline at 0.01 & 7.63 & 8.62 & 8.22 & 8.43 & 9.28 & 7.55 \\
\hline
\end{tabular}

While, Gaori was the greatest general combiner for plant height. In addition, the genotype, IEET1444 was good general combiners for improving number of panicles per plant, salinity tolerance index and grain yield/plant. While, Wab 880SG-33 was the best general combiner for 1000-grain weight and number of filled grains per panicle. The results revealed that among the studied parents, Suweon 345 followed by Wab 880SG-33 and Sakha 105 were the best general combiners for sterility\%. Moreover, IEET1444 and Gz1368-S-5-4 were the best general combiners for grain yield /plant. Similar results were obtained previously by Shehata, (2004), Verma and Srivastava (2004), Soroush and Moumeni (2006). El Abd et al. (2007), Viswanathan Satheesh and Thiyagarajan (2008) and Muthuramu et al., (2010) and ElNaem (2010).

\section{Specific combining ability effects:}

High specific combining ability effects were caused by the dominance and interaction or epistatic effects (non-fixable genes) that existed between the crossed parents. The same can be used as an index to determine the usefulness of a particular cross-combination in the exploitation of heterosis. As shown in Table 2, under normal conditions, 18 crosses out of 39 cross combinations possessed significant desirable SCA effects for number of days to $50 \%$ heading, the highest estimated negative value $(-14.18)$ was recorded in Suweon 345 x Sakha 104 rice hybrid followed by Gaori x Sakha 104 
Sultan, M. S. et al.

(-12.96) and BG 34-8 X Giza 178 (-11.82). Furthermore, the hybrids, Giza $182 \times$ Giza 178 and Suweon 345 x Sakha 102 were the best cross combinations to improve shortness of plant height.

Table 2: Estimates of specific combining ability effects for yield and its related traits in $\mathrm{f} 1$ crosses

\begin{tabular}{|c|c|c|c|c|c|c|}
\hline \multirow{2}{*}{ Genotypes } & \multicolumn{3}{|c|}{ Days to $50 \%$ heading } & \multicolumn{3}{|c|}{ Plant height (cm) } \\
\hline & $\mathbf{N}$ & S1 & S2 & $\mathbf{N}$ & S1 & S2 \\
\hline Wab 880 SG -33 x Sakha 102 & $-2.33^{\star \star}$ & $9.03^{\star \star}$ & $9.77^{\star \star}$ & $-2.69^{\star \star}$ & $-7.86^{\star \star}$ & 1.26 \\
\hline Wab 880SG -33 x Sakha 104 & $8.15^{\star \star}$ & $-7.21^{\star \star}$ & $-7.54^{\star \star}$ & 1.08 & $15.27^{\star \star}$ & -0.21 \\
\hline Wab 880SG -33 x Giza 178 & $-5.82^{* *}$ & $-1.82^{*}$ & $-2.23^{\star *}$ & $1.62^{*}$ & $-7.40^{\star \star}$ & -1.05 \\
\hline Wab 880-p1 $\quad$ x Sakha 102 & $2.67^{* *}$ & $12.47^{* *}$ & $11.77^{* *}$ & $6.64^{* *}$ & $-3.09^{\star *}$ & $-6.41^{* *}$ \\
\hline x Sakha 104 & $2.15^{\star \star}$ & $-12.43^{\star \star}$ & $-7.54^{\star \star}$ & $-2.59^{\star \star}$ & $6.04^{\star \star}$ & 1.13 \\
\hline x Giza 178 & $-4.82^{\star \star}$ & -0.04 & $-4.23^{\star *}$ & $-4.05^{\star *}$ & $-2.96^{\star \star}$ & $5.28^{* *}$ \\
\hline x Sakha 102 & $1.56^{\star}$ & $9.03^{\star *}$ & $7.10^{\star \star}$ & $14.97^{\star \star}$ & $14.80^{\star \star}$ & $7.92^{\star \star}$ \\
\hline x Sakha 104 & $-1.62^{*}$ & -0.21 & $1.80^{*}$ & $2.74^{\star \star}$ & $9.93^{* *}$ & $11.46^{\star *}$ \\
\hline x Giza 178 & 0.07 & $-8.82^{\star \star}$ & $-8.90^{\star *}$ & $-17.72^{* *}$ & $-24.74^{* *}$ & $-19.39^{* *}$ \\
\hline x Sakha 102 & $11.22^{\star \star}$ & $-2.97^{* *}$ & $-2.56^{\star *}$ & $8.31^{\star *}$ & $-3.86^{\star *}$ & $-2.41^{* *}$ \\
\hline x Sakha 104 & $-12.96^{\star *}$ & $-11.21^{\star *}$ & $-14.87^{* *}$ & $-4.92^{\star \star}$ & $-4.74^{\star \star}$ & $-4.87^{\star *}$ \\
\hline x Giza 178 & $1.74^{*}$ & $14.18^{* *}$ & $17.44^{* *}$ & $-3.39^{\star *}$ & $8.60^{* *}$ & $7.28^{* *}$ \\
\hline x Sakha 102 & 0.33 & $-11.53^{\star \star}$ & $-2.23^{\star \star}$ & -1.36 & $-2.09^{\star \star}$ & 0.92 \\
\hline x Sakha 104 & $-4.18^{\star \star}$ & $-2.43^{* \star}$ & $2.46^{\star \star}$ & $-1.59^{*}$ & $-4.62^{\star \star}$ & $-9.54^{\star *}$ \\
\hline x Giza 178 & $3.85^{\star *}$ & $13.96^{\star *}$ & -0.23 & $2.95^{\star *}$ & $6.71^{\star *}$ & $8.62^{* *}$ \\
\hline x Sakha 102 & $-3.00^{* *}$ & $-3.64^{* *}$ & $-5.01^{* *}$ & $2.31^{\star *}$ & $11.14^{* *}$ & $4.59^{\star *}$ \\
\hline x Sakha 104 & $7.49^{\star \star}$ & $15.13^{* *}$ & $13.02^{* *}$ & $20.08^{* *}$ & $9.27^{* *}$ & $19.13^{* *}$ \\
\hline x Giza 178 & $-4.49^{* *}$ & $-11.49^{* *}$ & $-8.01^{* *}$ & $-22.39^{* *}$ & $-20.40^{* *}$ & $-23.72^{* *}$ \\
\hline x Sakha 102 & 0.67 & 0.80 & $-3.90^{\star *}$ & $2.31^{\star \star}$ & -1.53 & $-4.08^{\star \star}$ \\
\hline x Sakha 104 & $-1.85^{\star}$ & $4.57^{\star \star}$ & $7.46^{\star \star}$ & $-8.92^{\star \star}$ & $-7.40^{\star \star}$ & $-10.54^{\star \star}$ \\
\hline x Giza 178 & 1.18 & $-5.38^{* *}$ & $-3.56^{\star *}$ & $6.62^{\star \star}$ & $8.93^{* *}$ & $14.62^{* \star}$ \\
\hline x Sakha 102 & $2.00^{*}$ & $-8.53^{\star *}$ & $3.44^{\star *}$ & $2.20^{\star \star}$ & -0.86 & $-3.08^{\star *}$ \\
\hline x Sakha 104 & $-2.51^{* *}$ & -1.43 & 0.13 & -0.70 & $-10.74^{* *}$ & $-9.54^{* *}$ \\
\hline x Giza 178 & 0.51 & $9.96^{\star *}$ & $-3.56^{\star \star}$ & -1.50 & $11.60^{\star *}$ & $12.62^{\star \star}$ \\
\hline x Sakha 102 & $-4.67^{* *}$ & $-4.64^{* *}$ & $-8.90^{* *}$ & $-18.80^{* *}$ & $-3.86^{\star *}$ & $3.92^{\star *}$ \\
\hline x Sakha 104 & $-14.18^{\star \star}$ & $-8.87^{\star \star}$ & $-7.21^{\star \star}$ & $-10.70^{\star \star}$ & $-9.74^{\star \star}$ & $-8.54^{\star \star}$ \\
\hline x Giza 178 & $18.85^{\star \star}$ & $13.51^{\star *}$ & $16.10^{\star *}$ & $29.50^{* *}$ & $13.60^{\star *}$ & $4.62^{\star \star}$ \\
\hline Gz 1368-S-5-4 & -0.33 & 1.58 & $-2.23^{\star \star}$ & $-4.03^{\star \star}$ & $-1.86^{*}$ & -0.41 \\
\hline Gz 1368-S-5-4 x Sakha 104 & $10.15^{\star \star}$ & $13.35^{\star *}$ & $11.46^{\star *}$ & $6.74^{\star *}$ & $3.27^{\star \star}$ & $11.13^{\star *}$ \\
\hline Gz 1368-S-5-4 & $-9.82^{\star *}$ & $-14.93^{* *}$ & $-9.23^{* *}$ & $-2.72^{\star *}$ & -1.40 & $-10.72^{* *}$ \\
\hline x Sakha 102 & $-3.33^{\star *}$ & $10.03^{\star *}$ & $4.66^{* *}$ & -0.36 & $-6.20^{* *}$ & $-3.74^{\star *}$ \\
\hline x Sakha 104 & $15.15^{\star \star}$ & $7.80^{\star *}$ & $6.68^{\star \star}$ & $14.41^{\star \star}$ & $17.93^{\star \star}$ & $9.80^{\star \star}$ \\
\hline x Giza 178 & $-11.82^{\star \star}$ & $-17.82^{\star \star}$ & $-11.34^{\star \star}$ & $-14.05^{\star \star}$ & $-11.74^{\star \star}$ & $-6.05^{\star \star}$ \\
\hline x Sakha 102 & $-2.67^{* *}$ & -0.64 & $-3.34^{\star *}$ & $-4.47^{* *}$ & $7.14^{\star *}$ & $5.26^{\star *}$ \\
\hline x Sakha 104 & $3.82^{\star *}$ & $4.13^{\star \star}$ & 0.68 & $-2.37^{\star *}$ & $-7.74^{\star \star}$ & $-5.21^{\star *}$ \\
\hline x Giza 178 & -1.15 & $-3.49^{* *}$ & $2.66^{* *}$ & $6.84^{\star *}$ & 0.60 & -0.05 \\
\hline x Sakha 102 & $-2.11^{* *}$ & $-10.97^{* *}$ & $-8.56^{\star *}$ & $-5.03^{* *}$ & $-1.86^{\star}$ & $-3.74^{\star *}$ \\
\hline x Sakha 104 & $-9.62^{\star *}$ & -1.21 & $-6.54^{\star \star}$ & $-13.26^{\star \star}$ & $-16.74^{\star \star}$ & $-4.21^{\star \star}$ \\
\hline x Giza 178 & $11.74^{\star \star}$ & $12.18^{\star \star}$ & $15.10^{\star *}$ & $18.28^{\star \star}$ & $18.60^{\star \star}$ & $7.95^{\star \star}$ \\
\hline S.E $\left(S_{i j}\right)$ & 0.77 & 0.81 & 0.81 & 0.76 & 0.78 & 0.79 \\
\hline S.E $\left(S_{i j}-S_{k l}\right)$ & 1.08 & 1.14 & 1.15 & 1.08 & 1.10 & 1.12 \\
\hline LS D at 0.05 & 1.54 & 1.62 & 1.62 & 1.52 & 1.56 & 1.58 \\
\hline at 0.01 & 2.05 & 2.15 & 2.15 & 2.02 & 2.07 & 2.10 \\
\hline
\end{tabular}


While, Suweon 345 x Sakha 102 and Sakha 105 x Giza 178 were the best cross combinations for improving number of panicles per plant. In addition, Sakha $106 \times$ Sakha 102 and Agami M1 x Giza 178 were the greatest cross combinations for 1000- grain weight. The results indicated that among the studied crosses, BG 34-8 x Giza 178 the best cross combination for number of filled grains/panicle, sterility\% and grain yield /plant. Moreover, Wab 880SG-33 x Sakha 104 was the best cross combination for grain yield /plant under normal conditions. The superiority of these crosses may be due to complementary and duplicate type of gene interactions. Hence, these hybrids are expected to produce desirable segregates and could be exploited successfully in breeding programs.

On the other side, under level (S1) of salinity conditions, the cross combination BG34-8 x Giza178 the best cross combinations for number of days to $50 \%$ heading, number of filled grains/panicle and sterility $\%$. While, the best cross combinations for plant height were IEET $1444 \times$ Giza 178, Giza $182 \times$ Giza 178 and Yabani lulu $\times$ Sakha 104. The cross combinations, Suweon 345 x Giza 178, IEET1444 x Sakha 104 and Agami M1 x Giza 178 showed higher positive SCA effect for number of panicles/plant. Four crosses namely, Wab 880SG-33 x Sakha 104, Yabani lulu x Giza 178, Suweon 345 x Sakha 102 and BG34-8 x Sakha 102 exhibited highly significant and positive SCA effects for 1000- grain weight. The results indicated that among the studied crosses, IEET1444 x Sakha 104 was the best cross combinations for salinity tolerance index and grain yield /plant. The crosses which gave the highest values of these traits could be used in breeding program for improving these traits. Similar findings were reported earlier by El Abd et al. (2003), Verma and Srivastava (2004), Dhakar and vinit (2006), Hariprasanna et al. (2006), El-Naem (2010) and Negm (2011).

In case of, the level (S2) of salinity condition, 21 out of the 39 hybrid combinations showed significant, highly significant and negative SCA effects for No. of days to $50 \%$ heading. Giza $182 \times$ Giza 178 was the best cross combination for sterility\%, number of filled grains/panicle and plant height. The best specific combinations for number of filled grains/panicle and number of panicles/plant was Suweon 345 x Giza 178. Three crosses namely, Yabani lulu x Sakha 102, Sakha 106 x Sakha 104 and IEET1444 x Sakha 104 exhibited highly significant and positive SCA effects for 1000-grain weight.

The cross combination, BG 34-8 x Giza 178 showed higher SCA effects for number of filled grains/panicle and sterility\%. Results in the Table 2 indicated that significant and highly significant positive estimates of specific combining ability were illustrated for 9 crosses for salinity tolerance index, these estimates varied from 1.39 to 16.84 $\%$ for Gaori x Sakha 104, IEET1444 x Sakha 104, respectively. Ten crosses showed significant and highly significant desirable SCA effects for grain yield /plant, highest estimated values were recorded in IEET1444 $\mathrm{x}$ Sakha 104 (10.18) followed by Wab880SG-33 x Sakha 104 (5.10) and Sakha 105 x Giza 178 (3.71). 
Sultan, M. S. et al.

Table 2 : Cont.

\begin{tabular}{|c|c|c|c|c|c|c|}
\hline \multirow{2}{*}{ Genotypes } & \multicolumn{3}{|c|}{ No. of panicles/plant } & \multicolumn{3}{|c|}{ 1000-grain weight (g) } \\
\hline & $\mathbf{N}$ & S1 & S2 & $\mathbf{N}$ & S1 & S2 \\
\hline Wab 880 SG -33 x Sakha 102 & $2.06^{\star *}$ & $-2.31^{\star \star}$ & 1.08 & -0.03 & -0.13 & 0.16 \\
\hline Wab 880SG -33 x Sakha 104 & -0.66 & $4.08^{\star \star}$ & 0.31 & -0.03 & $0.37^{* *}$ & 0.17 \\
\hline Wab 880SG -33 x Giza 178 & -1.40 & $-1.77^{*}$ & -1.39 & $0.06^{* *}$ & $-0.24^{*}$ & $-0.34^{\star *}$ \\
\hline Wab 880-p1 x Sakha 102 & -0.61 & -1.31 & 0.08 & 0.02 & $-0.27^{\star \star}$ & -0.05 \\
\hline x Sakha 104 & $-4.33^{* *}$ & $4.08^{\star \star}$ & $3.31^{* *}$ & $0.08^{\star \star}$ & 0.12 & 0.06 \\
\hline x Giza 178 & $4.93^{\star \star}$ & $-2.77^{\star \star}$ & $-3.39^{\star *}$ & $-0.09^{* *}$ & 0.15 & 0.00 \\
\hline x Sakha 102 & $2.39^{* *}$ & $3.69^{* *}$ & $3.74^{\star *}$ & 0.04 & 0.07 & -0.10 \\
\hline x Sakha 104 & $-2.33^{\star \star}$ & $7.08^{\star *}$ & $5.97^{\star *}$ & $0.10^{\star *}$ & $0.22^{*}$ & $0.31^{* *}$ \\
\hline x Giza 178 & -0.07 & $-10.77^{\star *}$ & $-9.72^{\star \star}$ & $-0.14^{\star *}$ & $-0.29^{* *}$ & $-0.22^{*}$ \\
\hline x Sakha 102 & $-4.27^{\star \star}$ & -1.31 & $-1.59^{*}$ & 0.00 & $-0.25^{\star \star}$ & $-0.24^{\star}$ \\
\hline x Sakha 104 & $2.01^{\star *}$ & $-3.92^{\star \star}$ & -1.36 & $0.27^{\star \star}$ & 0.13 & 0.05 \\
\hline x Giza 178 & $2.27^{\star \star}$ & $5.23^{\star \star}$ & $2.95^{\star *}$ & $-0.27^{* *}$ & 0.13 & 0.19 \\
\hline x Sakha 102 & $-2.61^{* *}$ & -1.31 & $-1.92^{*}$ & 0.00 & 0.10 & -0.06 \\
\hline x Sakha 104 & $1.68^{*}$ & $2.08^{* *}$ & $2.31^{* *}$ & -0.03 & -0.02 & -0.13 \\
\hline x Giza 178 & 0.93 & -0.77 & -0.39 & 0.03 & -0.08 & 0.19 \\
\hline x Sakha 102 & 0.28 & 0.36 & $2.41^{\star *}$ & $0.09^{* *}$ & 0.11 & 0.14 \\
\hline x Sakha 104 & $3.90^{\star \star}$ & $5.74^{\star \star}$ & $3.64^{\star *}$ & -0.01 & 0.09 & 0.00 \\
\hline x Giza 178 & $-4.18^{\star \star}$ & $-6.10^{\star \star}$ & $-6.05^{\star \star}$ & $-0.08^{* \star}$ & $-0.19^{*}$ & -0.14 \\
\hline x Sakha 102 & $-2.61^{* *}$ & $-2.31^{* *}$ & $-3.59^{\star \star}$ & $0.34^{* *}$ & -0.17 & $-0.62^{* *}$ \\
\hline x Sakha 104 & 0.68 & -0.92 & 0.64 & $-0.15^{\star \star}$ & $0.24^{*}$ & $0.52^{\star *}$ \\
\hline x Giza 178 & $1.93^{\star}$ & $3.23^{\star \star}$ & $2.95^{\star *}$ & $-0.19^{\star \star}$ & -0.07 & 0.09 \\
\hline x Sakha 102 & $-7.61^{\star *}$ & $2.03^{\star *}$ & $2.08^{* *}$ & $-0.26^{* *}$ & -0.12 & -0.11 \\
\hline x Sakha 104 & -1.33 & -0.59 & $-1.69^{*}$ & $0.10^{\star *}$ & 0.13 & -0.04 \\
\hline x Giza 178 & $8.93^{\star \star}$ & -1.44 & -0.39 & $0.16^{\star \star}$ & -0.01 & 0.14 \\
\hline x Sakha 102 & $16.39^{* \star}$ & $-5.31^{* *}$ & $-4.26^{* \star}$ & $-0.24^{* *}$ & $0.34^{\star *}$ & 0.08 \\
\hline x Sakha 104 & $-14.33^{\star *}$ & $-6.92^{\star \star}$ & $-6.03^{\star \star}$ & $0.26^{* \star}$ & $-0.41^{* *}$ & -0.17 \\
\hline x Giza 178 & $-2.07^{\star \star}$ & $12.23^{\star \star}$ & $10.28^{* \star}$ & -0.02 & 0.08 & 0.09 \\
\hline Gz 1368-S-5-4 & $-2.61^{\star *}$ & $5.03^{\star *}$ & $4.74^{* *}$ & $0.07^{* *}$ & 0.08 & 0.09 \\
\hline Gz 1368-S-5-4 x Sakha 104 & $3.68^{\star \star}$ & -0.59 & -1.03 & $-0.07^{\star *}$ & 0.01 & -0.12 \\
\hline Gz 1368-S-5-4 x Giza 178 & -1.07 & $-4.44^{* *}$ & $-3.72^{\star \star}$ & -0.01 & -0.09 & 0.02 \\
\hline x Sakha 102 & -1.27 & 1.36 & 1.08 & $0.24^{\star \star}$ & $0.30^{\star *}$ & 0.14 \\
\hline x Sakha 104 & $3.01^{\star *}$ & 0.74 & $2.31^{* *}$ & $-0.30^{* *}$ & $-0.41^{* *}$ & $-0.25^{*}$ \\
\hline x Giza 178 & $-1.74^{*}$ & $-2.10^{\star \star}$ & $-3.39^{* \star}$ & $0.06^{* *}$ & 0.12 & 0.11 \\
\hline x Sakha 102 & 1.06 & 0.36 & $-2.92^{\star \star}$ & $-0.13^{\star \star}$ & 0.01 & 0.14 \\
\hline x Sakha 104 & $4.34^{* *}$ & $-7.26^{\star *}$ & $-4.69^{\star \star}$ & $-0.17^{* *}$ & -0.16 & $-0.30^{* *}$ \\
\hline x Giza 178 & $-5.40^{* *}$ & $6.90^{\star *}$ & $7.62^{* *}$ & $0.30^{* *}$ & 0.14 & 0.17 \\
\hline x Sakha 102 & -0.61 & 1.03 & -0.92 & $-0.13^{\star \star}$ & -0.07 & $0.42^{* *}$ \\
\hline x Sakha 104 & $3.68^{\star \star}$ & $-3.59^{\star \star}$ & $-3.69^{\star \star}$ & $-0.07^{\star \star}$ & $-0.29^{*}$ & -0.13 \\
\hline x Giza 178 & $-3.07^{* *}$ & $2.56^{\star *}$ & $4.62^{\star \star}$ & $0.20^{* *}$ & $0.35^{\star *}$ & $-0.30^{\star *}$ \\
\hline S.E $\left(S_{i i}\right)$ & 0.74 & 0.76 & 0.75 & 0.02 & 0.09 & 0.10 \\
\hline S.E $\left(S_{i j}-S_{k l}\right)$ & 1.05 & 1.08 & 1.06 & 0.03 & 0.13 & 0.14 \\
\hline LS D at 0.05 & 1.48 & 1.52 & 1.50 & 0.04 & 0.18 & 0.2 \\
\hline at 0.01 & 1.97 & 2.02 & 2.00 & 0.05 & 0.24 & 0.27 \\
\hline
\end{tabular}

*and ${ }^{* \star}$ significant at 0.05 and 0.01 probability levels, respectively. Abbreviations: $N$ normal conditions, S1, first level of salinity $(4000 \mathrm{ppm})$, S2, second level of salinity (6000ppm) 
Table 2 : Cont.

\begin{tabular}{|c|c|c|c|c|c|}
\hline \multirow{2}{*}{ Genotype } & \multicolumn{3}{|c|}{ No. of filled grains/panicle } & \multicolumn{2}{|c|}{ Salinity index } \\
\hline & $\mathbf{N}$ & S1 & S2 & S1 & S2 \\
\hline Wab 880 SG -33 x & $3.39^{*}$ & 0.38 & $-14.92^{\star *}$ & -0.41 & -0.65 \\
\hline Wab 880SG -33 x Sakha 104 & $40.39^{* *}$ & $49.79^{* *}$ & $26.03^{\star \star}$ & $1.42^{*}$ & 0.70 \\
\hline Wab 880SG -33 x Giza 178 & $-43.77^{\star *}$ & $-50.16^{\star *}$ & $-11.12^{* *}$ & -1.01 & -0.05 \\
\hline Wab 880-p1 $\quad$ x Sakha 102 & $10.39^{\star *}$ & $-13.40^{*}$ & $4.53^{\star *}$ & $-12.27^{* \star}$ & $-2.53^{\star \star}$ \\
\hline x Sakha 104 & $3.39^{*}$ & $18.68^{\star *}$ & $25.15^{\star \star}$ & $20.89^{\star *}$ & $6.08^{\star \star}$ \\
\hline x Giza 178 & $-13.77^{\star \star}$ & $-5.27^{\star \star}$ & $-29.68^{* *}$ & $-8.62^{\star \star}$ & $-3.55^{\star *}$ \\
\hline x Sakha 102 & $7.72^{\star *}$ & $-26.07^{* *}$ & $-17.92^{* *}$ & $-19.40^{* *}$ & $-9.40^{* *}$ \\
\hline x Sakha 104 & $-28.28^{* \star}$ & $46.01^{\star *}$ & $14.37^{\star \star}$ & $33.15^{\star \star}$ & $16.84^{\star *}$ \\
\hline x Giza 178 & $20.56^{\star *}$ & $-19.94^{* *}$ & $3.55^{\star *}$ & $-13.75^{* *}$ & $-7.44^{* *}$ \\
\hline x Sakha 102 & $10.39^{\star *}$ & $2.38^{* *}$ & $6.09^{* *}$ & $-4.67^{\star *}$ & -0.60 \\
\hline x Sakha 104 & $-36.62^{\star *}$ & $4.45^{\star \star}$ & $11.70^{\star \star}$ & -0.24 & $1.39^{*}$ \\
\hline x Giza 178 & $26.23^{* *}$ & $-6.83^{\star *}$ & $-17.79^{* *}$ & $4.92^{\star *}$ & -0.79 \\
\hline x Sakha 102 & $14.05^{\star \star}$ & $8.49^{\star \star}$ & $21.53^{\star *}$ & $-8.27^{\star \star}$ & $-2.95^{\star *}$ \\
\hline x Sakha 104 & $37.05^{\star *}$ & $6.56^{\star *}$ & $-11.52^{* *}$ & -0.33 & $-1.66^{\star *}$ \\
\hline x Giza 178 & $-51.10^{* *}$ & $-15.05^{\star *}$ & $-10.01^{* *}$ & $8.60^{* *}$ & $4.61^{* *}$ \\
\hline x Sakha 102 & $-19.95^{* *}$ & $-12.51^{* *}$ & $-7.47^{\star \star}$ & $14.62^{* *}$ & $3.98^{* *}$ \\
\hline x Sakha 104 & $-47.95^{\star \star}$ & $-8.44^{\star \star}$ & -1.52 & $-9.47^{\star \star}$ & $-4.16^{\star \star}$ \\
\hline x Giza 178 & $67.90^{\star *}$ & $20.95^{\star \star}$ & $8.99^{\star *}$ & $-5.15^{\star \star}$ & 0.19 \\
\hline x Sakha 102 & $-6.95^{\star *}$ & $18.60^{* *}$ & $9.64^{* *}$ & $-4.90^{* *}$ & 0.96 \\
\hline x Sakha 104 & $39.05^{\star *}$ & $2.68^{* *}$ & -1.41 & 0.93 & $-1.69^{* *}$ \\
\hline x Giza 178 & $-32.10^{* *}$ & $-21.27^{* *}$ & $-8.23^{* *}$ & $3.97^{\star *}$ & 0.74 \\
\hline x Sakha 102 & $37.05^{* *}$ & $4.60^{* *}$ & $10.31^{* *}$ & $4.03^{\star *}$ & $4.47^{* *}$ \\
\hline x Sakha 104 & $22.05^{\star *}$ & $-6.99^{\star *}$ & $-2.41^{* *}$ & $-5.12^{\star *}$ & $-4.60^{* *}$ \\
\hline x Giza 178 & $-59.10^{\star \star}$ & $2.39^{\star \star}$ & $-7.90^{\star \star}$ & 1.09 & 0.13 \\
\hline x Sakha 102 & $14.39^{\star *}$ & $9.60^{* *}$ & $3.31^{\star *}$ & $-3.53^{\star *}$ & $1.45^{\star}$ \\
\hline x Sakha 104 & -1.62 & $-31.66^{\star \star}$ & $-13.74^{* *}$ & $-4.85^{\star \star}$ & -0.11 \\
\hline Suweon 345 & $-12.77^{* *}$ & $22.06^{\star *}$ & $10.44^{* *}$ & $8.38^{\star \star}$ & $-1.34^{*}$ \\
\hline Gz 1368-S-5-4 & $-48.28^{\star *}$ & $-12.07^{* *}$ & $-2.69^{\star *}$ & $7.25^{\star *}$ & 0.36 \\
\hline Gz 1368-S-5-4 & $-12.28^{* *}$ & $-34.33^{\star *}$ & $-20.74^{\star *}$ & $-4.47^{\star *}$ & -0.63 \\
\hline Gz 1368-S-5-4 x Giza 178 & $60.56^{\star \star}$ & $46.39^{\star *}$ & $23.44^{\star \star}$ & $-2.78^{\star \star}$ & 0.28 \\
\hline x Sakha 102 & $-17.28^{* *}$ & $-18.96^{\star *}$ & $-24.80^{* *}$ & $11.15^{\star *}$ & $6.26^{* *}$ \\
\hline x Sakha 104 & $-80.28^{\star *}$ & $-38.21^{\star *}$ & $-24.86^{\star *}$ & $-5.35^{\star \star}$ & 0.56 \\
\hline x Giza 178 & $97.56^{\star *}$ & $57.17^{* *}$ & $49.66^{\star *}$ & $-5.80^{* *}$ & $-6.82^{* *}$ \\
\hline x Sakha 102 & $-39.62^{\star \star}$ & $33.82^{\star \star}$ & $3.86^{*}$ & $19.85^{\star \star}$ & $1.58^{*}$ \\
\hline x Sakha 104 & $69.39^{* *}$ & $-7.44^{\star *}$ & 1.15 & $-11.10^{* *}$ & $-1.94^{\star \star}$ \\
\hline x Giza 178 & $-29.77^{\star *}$ & $-26.39^{\star \star}$ & $-5.01^{\star \star}$ & $-8.74^{\star *}$ & 0.36 \\
\hline x Sakha 102 & $34.72^{\star *}$ & $5.15^{\star *}$ & $8.53^{* *}$ & $-3.44^{* *}$ & $-2.91^{\star *}$ \\
\hline x Sakha 104 & $-30.44^{\star *}$ & $-4.05^{\star *}$ & $-6.34^{\star *}$ & $-15.46^{\star \star}$ & $-10.78^{\star *}$ \\
\hline S.E $\left(\mathrm{S}_{\mathrm{ij}}\right)$ & 0.84 & 0.89 & 0.90 & 0.60 & 0.62 \\
\hline S.E $\left(S_{i j}-S_{k l}\right)$ & 1.19 & 1.25 & 1.27 & 0.85 & 0.88 \\
\hline LS D at 0.05 & 1.68 & 1.78 & 1.8 & 1.2 & 1.24 \\
\hline \begin{tabular}{|l|} 
at 0.01 \\
\end{tabular} & 2.23 & 2.37 & 2.39 & 1.60 & 1.65 \\
\hline
\end{tabular}

*and ${ }^{* *}$ significant at 0.05 and 0.01 probability levels, respectively. Abbreviations: $N$ normal conditions, S1, first level of salinity $(4000 \mathrm{ppm}), \mathrm{S} 2$, second level of salinity (6000ppm) 
Sultan, M. S. et al.

Table 2 : Cont.

\begin{tabular}{|c|c|c|c|c|c|c|}
\hline \multirow{2}{*}{ Genotypes } & \multicolumn{3}{|c|}{ Sterility \% } & \multicolumn{3}{|c|}{ Grain yield/plant (g) } \\
\hline & $\mathbf{N}$ & S1 & S2 & $\mathbf{N}$ & S1 & S2 \\
\hline Wab 880 SG -33 x Sakha 102 & -1.42 & $-3.31^{\star \star}$ & $7.90^{\star \star}$ & $-6.21^{\star \star}$ & $-2.44^{*}$ & -1.39 \\
\hline Wab 880SG -33 x Sakha 104 & $-3.22^{* *}$ & $-8.05^{\star *}$ & $-13.77^{\star *}$ & $28.48^{\star \star}$ & $9.52^{* *}$ & $5.10^{* *}$ \\
\hline Wab 880SG -33 x Giza 178 & $4.64^{\star \star}$ & $11.36^{\star \star}$ & $5.88^{\star \star}$ & $-22.26^{\star \star}$ & $-7.08^{\star \star}$ & $-3.71^{\star \star}$ \\
\hline Wab 880-p1 x Sakha 102 & $16.66^{* *}$ & $4.19^{\star *}$ & $-4.12^{\star *}$ & $15.91^{\star \star}$ & $-2.08^{*}$ & 1.25 \\
\hline x Sakha 104 & $-4.09^{\star \star}$ & $-3.97^{\star \star}$ & $-15.44^{\star \star}$ & $-8.20^{* *}$ & $9.92^{\star \star}$ & $2.30^{\star \star}$ \\
\hline x Giza 178 & $-12.58^{\star *}$ & -0.22 & $19.56^{* *}$ & $-7.71^{\star \star}$ & $-7.85^{\star \star}$ & $-3.55^{\star \star}$ \\
\hline x Sakha 102 & -0.03 & $13.27^{\star \star}$ & $13.80^{\star *}$ & $6.77^{\star *}$ & $-11.63^{* \star}$ & $-5.44^{* *}$ \\
\hline x Sakha 104 & $13.99^{\star \star}$ & $9.50^{\star *}$ & $2.93^{* *}$ & $-8.91^{* *}$ & $20.47^{* *}$ & $10.18^{\star \star}$ \\
\hline x Giza 178 & $-13.97^{* *}$ & $-22.77^{\star *}$ & $-16.73^{\star *}$ & $2.15^{*}$ & $-8.83^{* *}$ & $-4.74^{\star \star}$ \\
\hline x Sakha 102 & 0.60 & $-11.62^{\star \star}$ & $-23.23^{\star \star}$ & $7.64^{\star \star}$ & -0.05 & 0.81 \\
\hline x Sakha 104 & 0.93 & $1.84^{*}$ & $4.31^{\star *}$ & -1.73 & -0.89 & 0.80 \\
\hline x Giza 178 & -1.53 & $9.78^{\star *}$ & $18.92^{* *}$ & $-5.91^{\star *}$ & 0.94 & $-1.61^{*}$ \\
\hline x Sakha 102 & $-11.09^{\star \star}$ & $-10.53^{\star \star}$ & $-16.56^{\star \star}$ & $8.80^{\star \star}$ & -1.93 & -0.27 \\
\hline x Sakha 104 & $-13.25^{\star *}$ & $-17.42^{\star \star}$ & 0.25 & $4.61^{\star *}$ & 0.87 & -0.18 \\
\hline Giza 177 & $24.34^{\star \star}$ & $27.95^{\star \star}$ & $16.32^{* *}$ & $-13.41^{* *}$ & 1.07 & 0.44 \\
\hline x Sakha 102 & $-15.92^{* *}$ & $14.75^{\star *}$ & $16.49^{\star *}$ & $-11.56^{\star *}$ & $4.66^{* \star}$ & 0.77 \\
\hline x Sakha 104 & $32.53^{\star \star}$ & $15.53^{\star \star}$ & $6.51^{\star \star}$ & $19.40^{\star \star}$ & -1.25 & -0.58 \\
\hline x Giza 178 & $-16.61^{* *}$ & $-30.28^{\star *}$ & $-23.01^{* *}$ & $-7.84^{\star *}$ & $-3.41^{* *}$ & -0.19 \\
\hline x Sakha 102 & $-11.74^{\star *}$ & $-13.85^{\star \star}$ & $-16.66^{\star \star}$ & $-6.56^{\star *}$ & $-5.70^{\star \star}$ & $-2.17^{* \star}$ \\
\hline x Sakha 104 & $-16.37^{\star *}$ & $-15.44^{\star \star}$ & $-4.87^{\star \star}$ & $-2.09^{*}$ & 0.30 & -0.74 \\
\hline x Giza 178 & $28.11^{\star \star}$ & $29.29^{\star *}$ & $21.53^{\star *}$ & $8.65^{\star *}$ & $5.40^{\star \star}$ & $2.91^{\star \star}$ \\
\hline x Sakha 102 & $-7.43^{\star *}$ & $-4.98^{\star \star}$ & $-1.76^{*}$ & $-10.79^{* *}$ & -0.50 & 0.24 \\
\hline x Sakha 104 & $-8.65^{\star \star}$ & $-8.75^{\star \star}$ & $-6.78^{\star *}$ & $-5.93^{* *}$ & $-5.07 * *$ & $-3.94^{\star \star}$ \\
\hline x Giza 178 & $16.08^{\star \star}$ & $13.73^{\star \star}$ & $8.54^{\star \star}$ & $16.73^{\star \star}$ & $5.57^{\star \star}$ & $3.71^{\star \star}$ \\
\hline x Sakha 102 & $-5.77^{* *}$ & $-12.67^{\star \star}$ & $-5.27^{\star *}$ & $-10.89^{* \star}$ & $-2.95^{\star *}$ & 0.69 \\
\hline x Sakha 104 & $-6.63^{\star \star}$ & $-4.15^{\star *}$ & $-5.73^{* *}$ & $3.67^{\star \star}$ & $-5.62^{\star \star}$ & 0.01 \\
\hline Suweon 345 & $12.40^{* \star}$ & $16.82^{\star \star}$ & $11.7^{\star \star}$ & $7.23^{\star \star}$ & $8.58^{\star \star}$ & -0.70 \\
\hline Gz 1368-S-5-4 & $23.60^{* *}$ & $9.33^{* *}$ & $7.17^{* *}$ & $-19.97^{\star *}$ & $3.57^{\star *}$ & -1.11 \\
\hline Gz 1368-S-5-4 & $2.19^{*}$ & $21.24^{* *}$ & $19.46^{* *}$ & $-7.41^{* *}$ & $-6.30^{\star *}$ & $-2.36^{\star \star}$ \\
\hline Gz 1368-S-5-4 x Giza 178 & $-25.79^{\star *}$ & $-30.57^{\star \star}$ & $-26.62^{\star \star}$ & $27.38^{\star \star}$ & $2.73^{\star \star}$ & $3.47^{\star \star}$ \\
\hline x Sakha 102 & $13.12^{\star *}$ & $23.86 * 8$ & $31.03^{* *}$ & $7.30^{\star *}$ & $4.18^{\star *}$ & $3.04^{\star *}$ \\
\hline x Sakha 104 & $32.21^{\star \star}$ & $32.05^{* *}$ & $28.59^{* *}$ & $-28.95^{\star *}$ & $-10.36^{* *}$ & $-4.44^{* *}$ \\
\hline x Giza 178 & $-45.34^{\star *}$ & $-55.91^{\star \star}$ & $-59.62^{\star \star}$ & $21.65^{\star \star}$ & $6.18^{* \star}$ & 1.41 \\
\hline x $\quad$ Sakha 102 & $14.49^{\star \star}$ & $6.08^{\star \star}$ & $16.68^{* *}$ & $4.75^{\star \star}$ & $11.49^{\star \star}$ & $1.90^{*}$ \\
\hline x Sakha 104 & $-21.26^{\star *}$ & $-15.91^{* *}$ & $-20.10^{\star \star}$ & -1.06 & $-5.98^{\star *}$ & -1.48 \\
\hline x Giza 178 & $6.77^{* *}$ & $9.83^{* *}$ & $3.42^{* *}$ & $-3.70^{* *}$ & $-5.51^{\star *}$ & -0.42 \\
\hline x Sakha 102 & $-15.08^{\star *}$ & $-14.52^{\star \star}$ & $-25.46^{\star *}$ & $14.81^{\star \star}$ & $3.39^{\star *}$ & $1.68^{*}$ \\
\hline x Sakha 104 & $-8.40^{* *}$ & $-6.47^{\star *}$ & $4.65^{\star \star}$ & $8.13^{\star \star}$ & $-5.61^{\star \star}$ & $-4.67^{\star \star}$ \\
\hline x Giza 178 & $23.48^{* *}$ & $20.99^{* *}$ & $20.81^{* *}$ & $-22.94^{\star *}$ & $2.22^{*}$ & $2.99^{* *}$ \\
\hline S.E $\left(S_{i j}\right)$ & 0.80 & 0.90 & 0.86 & 0.88 & 0.97 & 0.79 \\
\hline S.E $\left(S_{i j}-S_{k l}\right)$ & 1.13 & 1.27 & 1.21 & 1.24 & 1.37 & 1.11 \\
\hline LS D at 0.05 & 1.6 & 1.8 & 1.72 & 1.76 & 1.94 & 1.58 \\
\hline at 0.01 & 2.13 & 2.39 & 2.29 & 2.34 & 2.58 & 2.10 \\
\hline
\end{tabular}

*and ${ }^{* *}$ significant at 0.05 and 0.01 probability levels, respectively. Abbreviations: $N$ normal conditions, S1, first level of salinity $(4000 \mathrm{ppm})$, S2, second level of salinity (6000ppm)

These results indicated that most of these crosses could be utilized for improving grain yield trait in rice breeding program The results obtained here concerning general and specific combining ability effects could indicate that the excellent crosses which showed desirable SCA effects were obtained from crossing good by good, good by low and low by 
low combiners. Consequently, it could be concluded that GCA effects of the parental varieties were generally unrelated to the specific combining ability effects of their respective crosses. This conclusion was also reported by Sedeek (2006) and Chakraborty et al. (2010).

\section{Estimation of genetic parameters and heritability:}

The estimates of, phenotypic variance $\left(\sigma^{2} \mathrm{P}\right)$, genotype variance $\left(\sigma^{2}\right.$ $G)$, environmental variance $\left(\sigma^{2} E\right)$, dominance genetic variance $\left(\sigma^{2} D\right)$, additive genetic variance $\left(\sigma^{2} A\right)$, broad sense heritability $\left(h^{2} b \%\right)$, narrow sense heritability ( $h^{2} n \%$ ), GCA to Sca ratio are presented in Table 3.

It is evident from Table 3 that the dominance genetic variance was greater than additive genetic variance for all the studied traits. The highest estimated values of environmental variance were recorded for number of filled grains/panicle followed by number of days to $50 \%$ heading under normal and two saline soil conditions. On the other hand, high estimates of broad sense heritability coupled with high genetic advance were noted for number of filled grains/panicle and sterility\% under all conditions, revealing substantial contribution of additive genetic variance in phenotypic expression, and indicating the effectiveness of selection in early generations to improve these traits. Falconer and Mackay (1996) demonstrated that the lower narrow sense heritability was caused by low additive effects and high dominant gene action. These results are in harmony with the combining ability analysis. Low estimate values of narrow sense heritability were exhibited for all the studied traits under all studied conditions, On the contrary, the lowest values were observed for number of filled grains/panicle and sterility\% .

Table 3 : Genetic parameters for some vegetative, yield and its components traits under normal and saline $(\mathrm{S} 1, \mathrm{~S} 2)$ soil conditions.

\begin{tabular}{|l|c|c|c|c|c|c|}
\hline \multirow{2}{*}{ Genetic Parameters } & \multicolumn{3}{|c|}{ Days to 50\% heading } & \multicolumn{3}{c|}{ Plant height (cm) } \\
\cline { 2 - 7 } & $\mathbf{N}$ & $\mathbf{S 1}$ & $\mathbf{S 2}$ & $\mathbf{N}$ & $\mathbf{S 1}$ & $\mathbf{S 2}$ \\
\hline Phenotypic Variance & 98.17 & 81.54 & 115.23 & 205.28 & 184.48 & 141.62 \\
\hline Genetic Variance & 92.47 & 75.23 & 108.78 & 199.61 & 178.54 & 135.41 \\
\hline Environment Variance & 5.70 & 6.31 & 6.45 & 5.67 & 5.94 & 6.21 \\
\hline Dominance Variance & 81.10 & 71.81 & 105.46 & 172.61 & 167.79 & 124.71 \\
\hline Additive Variance & 11.37 & 3.42 & 3.32 & 27.00 & 10.75 & 10.70 \\
\hline Broad sense of Heritability & 94.19 & 92.27 & 94.40 & 97.24 & 96.78 & 95.61 \\
\hline Narrow sense of Heritability & 11.58 & 4.19 & 2.88 & 13.15 & 5.83 & 7.55 \\
\hline Genetic Advance & 1922.55 & 1716.27 & 2087.50 & 2869.93 & 2707.89 & 2343.95 \\
\hline Ratio of gca Var. to sca Var. & 1.14 & 1.05 & 1.03 & 1.16 & 1.06 & 1.09 \\
\hline
\end{tabular}

Abbreviations: $\mathbf{N}$, normal conditions, S1, first level of salinity (4000ppm),S2, second level of salinity (6000ppm)

Moreover, the ratio of GCA and SCA variances was greater than unity for all the traits studied that revealed the preponderance of additive gene action and additive $\mathrm{x}$ additive type of gene interaction in the inheritance of such traits. Hammoud (2004) who found that narrow sense heritability estimates were lower than of broad sense for most characters studied indicating the importance of non-additive genetic variance in the inheritance of vegetative characters, subsequently selection procedures are preferred in the late generation. 
Sultan, M. S. et al.

Table 3 : Cont.

\begin{tabular}{|l|c|c|c|c|c|c|}
\hline \multirow{2}{*}{ Genetic Parameters } & \multicolumn{3}{|c|}{ No. of panicles/plant } & \multicolumn{3}{|c|}{1000 -grain weight (g) } \\
\cline { 2 - 7 } & $\mathrm{N}$ & $\mathrm{S} 1$ & $\mathrm{~S} 2$ & $\mathrm{~N}$ & $\mathrm{~S} 1$ & $\mathrm{~S} 2$ \\
\hline Phenotypic Variance & 39.83 & 34.66 & 27.36 & 0.07 & 0.1 & 0.11 \\
\hline Genetic Variance & 37.48 & 31.91 & 25.06 & 0.04 & 0.06 & 0.08 \\
\hline Environment Variance & 2.35 & 2.75 & 2.30 & 0.03 & 0.04 & 0.03 \\
\hline Dominance Variance & 35.38 & 31.33 & 24.93 & 0.03 & 0.05 & 0.06 \\
\hline Additive Variance & 2.10 & 0.58 & 0.13 & 0.01 & 0.01 & 0.01 \\
\hline Broad sense of Heritability & 94.11 & 92.06 & 91.60 & 63.84 & 60.58 & 72.09 \\
\hline Narrow sense of Heritability & 5.27 & 1.66 & 0.48 & 17.90 & 8.58 & 11.91 \\
\hline Genetic Advance & 1223.43 & 1116.55 & 987.04 & 34.64 & 39.40 & 48.70 \\
\hline Ratio of gca Var. to sca Var. & 1.06 & 1.02 & 1.01 & 1.39 & 1.17 & 1.20 \\
\hline
\end{tabular}

Abbreviations: N, normal conditions, S1, first level of salinity (4000ppm), S2, second level of salinity $(6000 \mathrm{ppm})$

Table 3 : Cont.

\begin{tabular}{|l|c|c|c|c|c|}
\hline \multirow{2}{*}{ Genetic Parameters } & \multicolumn{2}{|c|}{ No. of filled grains/panicle } & \multicolumn{2}{c|}{ Salinity index } \\
\cline { 2 - 6 } & $\mathrm{N}$ & $\mathrm{S} 1$ & $\mathrm{~S} 2$ & $\mathrm{~S} 1$ & $\mathrm{~S} 2$ \\
\hline Phenotypic Variance & 2474.19 & 724.16 & 431.55 & 193.9 & 47.31 \\
\hline Genetic Variance & 2466.77 & 702.65 & 422.43 & 188.78 & 42.93 \\
\hline Environment Variance & 7.42 & 21.51 & 9.12 & 5.12 & 4.38 \\
\hline Dominance Variance & 2453.19 & 681.99 & 410.13 & 187.03 & 41.58 \\
\hline Additive Variance & 13.58 & 20.66 & 12.30 & 1.75 & 1.35 \\
\hline Broad sense of Heritability & 99.70 & 97.03 & 97.89 & 97.36 & 90.74 \\
\hline Narrow sense of Heritability & 0.55 & 2.85 & 2.85 & 0.90 & 2.86 \\
\hline Genetic Advance & 10215.96 & 5378.87 & 4188.95 & 2792.76 & 1285.75 \\
\hline Ratio of gca Var. to sca Var. & 1.01 & 1.03 & 1.03 & 1.01 & 1.03 \\
\hline
\end{tabular}

Abbreviations: N, normal conditions, S1, first level of salinity (4000ppm),S2, second leve of salinity $(6000 \mathrm{ppm})$

Table 3 : Cont.

\begin{tabular}{|l|c|c|c|c|c|c|}
\hline \multirow{2}{*}{ Genetic Parameters } & \multicolumn{3}{|c|}{ Sterility \% } & \multicolumn{3}{c|}{ Grain yield/ plant (g) } \\
\cline { 2 - 7 } & $\mathrm{N}$ & $\mathrm{S} 1$ & $\mathrm{~S} 2$ & $\mathrm{~N}$ & $\mathrm{~S} 1$ & $\mathrm{~S} 2$ \\
\hline Phenotypic Variance & 477.22 & 318.7 & 357.11 & 366.14 & 65.81 & 16.38 \\
\hline Genetic Variance & 475.02 & 316.28 & 355.21 & 363.82 & 63.00 & 14.52 \\
\hline Environment Variance & 2.20 & 2.42 & 1.90 & 2.32 & 2.81 & 1.86 \\
\hline Dominance Variance & 466.93 & 313.26 & 342.76 & 288.82 & 61.90 & 14.18 \\
\hline Additive Variance & 8.09 & 3.03 & 12.46 & 75.00 & 1.10 & 0.34 \\
\hline Broad sense of Heritability & 99.54 & 99.24 & 99.47 & 99.37 & 95.74 & 88.63 \\
\hline Narrow sense of Heritability & 1.69 & 0.95 & 3.49 & 20.48 & 1.67 & 2.08 \\
\hline Genetic Advance & 4479.38 & 3649.66 & 3872.16 & 3916.77 & 1599.80 & 739.03 \\
\hline Ratio of gca Var. to sca Var. & 1.02 & 1.01 & 1.04 & 1.26 & 1.02 & 1.02 \\
\hline
\end{tabular}

Abbreviations: N, normal conditions, S1, first level of salinity (4000ppm), S2, second level of salinity $(6000 \mathrm{ppm})$

The results suggest that improvement in these traits may be obtained via heterosis breeding or by single plant selection in early generations following hybridization or intermitting of selected segregates through recurrent selection. Similar results were observed previously by Verma and Srivastava (2004), Bagheri et al. (2005), Saxena et al. (2005), Manickavelu et al. (2006) Pradhan et al. (2006) and Sharma et al. (2007).

Estimates of phenotypic correlation coefficients.

Phenotypic correlation coefficients among all possible pairs of the studied characters are presented in Tables 4-6. The results in table 4 
revealed that days to $50 \%$ heading was significantly and positive associated with each of plant height, 1000-grain weight and grain yield/plant . On the contrary, highly significant negative estimated of phenotypic correlation coefficients was observed between days to $50 \%$ heading and sterility \% under normal conditions. Plant height was significantly correlated with sterility\%. While, Ganapathy et al. (2007), reported that Plant height was positively and significantly correlated with grain yield/plant.

Table 4: Estimates of phenotypic correlation coefficients between grain yield/plant and its components under normal conditions.

\begin{tabular}{|l|c|c|c|c|c|c|}
\hline Characters & $\mathbf{1}$ & $\mathbf{2}$ & $\mathbf{3}$ & $\mathbf{4}$ & $\mathbf{5}$ & $\mathbf{6}$ \\
\hline 1- Days to 50\% heading & - & & & & & \\
\hline 2- Plant height (cm) & $0.233^{\star}$ & - & & & & \\
\hline 3- No. of panicles /plant & 0.138 & 0.098 & - & & & \\
\hline 4- No. of filled grains /plant & 0.163 & -0.133 & 0.103 & - & & \\
\hline 5-1000-grain weight (g) & $0.202^{*}$ & 0.063 & $-0.310^{\star *}$ & $-0.242^{*}$ & - & \\
\hline 6- Sterility (\%) & $-0.199^{\star}$ & $0.381^{\star *}$ & 0.133 & $-0.317^{\star *}$ & -0.074 & - \\
\hline 7- Grain yield/plant (g) & $0.214^{*}$ & 0.075 & $0.329^{\star *}$ & $0.278^{\star *}$ & $0.402^{\star *}$ & -0.069 \\
\hline
\end{tabular}

*, ** Significant and highly significant at 0.05 and 0.01 levels of probability, respectively.

On the other hand, under the first level of salinity conditions significant or highly significant positive estimated of phenotypic correlation coefficients were obtained between days to $50 \%$ heading and each of plant height and salinity index. On the other hand, significant or highly significant positive estimated of phenotypic correlation coefficients obtained between plant height and number of panicles per plant, salinity index, sterility \% and grain yield per plant. In the same time, number of panicle/plant gave significant and positive correlation with each of salinity index and sterility \% with values (0.226) and (0.236), respectively. Moreover, highly significant positive correlation coefficient was recorded between number of filled grains per panicle and salinity index (Table 5).

Table 5: Estimates of phenotypic correlation coefficients between grain yield/plant and its components under level S1 conditions.

\begin{tabular}{|l|c|c|c|c|c|c|c|}
\hline Characters & $\mathbf{1}$ & $\mathbf{2}$ & $\mathbf{3}$ & $\mathbf{4}$ & $\mathbf{5}$ & $\mathbf{6}$ & $\mathbf{7}$ \\
\hline 1- Days to 50\% heading & - & & & & & & \\
\hline 2- Plant height (cm) & $0.207^{\star}$ & - & & & & & \\
\hline 3- No. of panicles /plant & 0.144 & $0.386^{\star *}$ & - & & & & \\
\hline $\begin{array}{l}\text { 4- No. of filled grains } \\
\text { /plant }\end{array}$ & -0.087 & 0.152 & 0.082 & - & & & \\
\hline 5-1000-grain weight (g) & 0.094 & 0.068 & -0.050 & 0.087 & - & & \\
\hline 6- Salinity index (\%) & $0.289^{\star *}$ & $0.287^{\star *}$ & $0.226^{*}$ & $0.389^{* *}$ & 0.040 & - & \\
\hline 7- Sterility (\%) & -0.032 & $0.195^{*}$ & $0.236^{*}$ & $-0.232^{*}$ & 0.039 & -0.022 & - \\
\hline 8- Grain yield/plant (g) & 0.063 & $0.312^{\star *}$ & $0.396^{* *}$ & $0.409^{* *}$ & $0.305^{\star *}$ & 0.049 & $-0.444^{\star *}$ \\
\hline
\end{tabular}

${ }^{*},{ }^{\star \star}$ Significant and highly significant at 0.05 and 0.01 levels of probability, respectively.

Moreover, highly significant positive estimated of phenotypic correlation coefficients were obtained between days to $50 \%$ heading and 1000 - grain weight. While, highly significant positive estimated of phenotypic correlation coefficients obtained between plant height and number of panicles per plant, salinity index under S2 conditions (Table 6). Highly significant positive 
estimates of phenotypic correlation coefficients were obtained between no. of panicles/plant with grain yield /plant under all conditions. These findings were in close agreement with those reported earlier by Abd-Allah (2000) and Abd El-Lattef et al. (2008).

Table 6: Estimates of phenotypic correlation coefficients between grain yield/plant and its components under level S2 conditions.

\begin{tabular}{|l|c|c|c|c|c|c|c|}
\hline Characters & $\mathbf{1}$ & $\mathbf{2}$ & $\mathbf{3}$ & $\mathbf{4}$ & $\mathbf{5}$ & $\mathbf{6}$ & $\mathbf{7}$ \\
\hline 1- Days to 50\% heading & - & & & & & & \\
\hline 2- Plant height (cm) & 0.131 & - & & & & & \\
\hline 3- No.of panicles /plant & 0.090 & $0.382^{* *}$ & - & & & & \\
\hline $\begin{array}{l}\text { 4- No. of filled grains } \\
\text { plant }\end{array}$ & -0.178 & 0.101 & 0.172 & - & & & \\
\hline 5-1000-grain weight (g) & $0.264^{* *}$ & 0.078 & -0.053 & 0.032 & - & & \\
\hline 6- Salinity index (\%) & 0.126 & $0.244^{* *}$ & 0.185 & $0.437^{* *}$ & -0.053 & - & \\
\hline 7- Sterility (\%) & -0.176 & 0.080 & 0.160 & -0.016 & -0.088 & -0.035 & - \\
\hline 8- Grain yield/plant (g) & -0.044 & 0.155 & $0.252^{* \star}$ & $0.387^{* *}$ & $0.316^{* *}$ & 0.135 & $-0.255^{\star *}$ \\
\hline
\end{tabular}

*, ** Significant and highly significant at 0.05 and 0.01 levels of probability, respectively.

Highly significant positive correlation coefficient was recorded between number of filled grains per panicle and grain yield/plant under all conditions. Regarding to 1000-grain weight, results emphasizes that it was positively and highly significantly phenotypically correlated with grain yield/plant under all conditions.

These results were in harmony with those of Abd-Allah (2000) and Abd El-Lattef et al. (2008). They reported that 1000-grain weight was positive correlated with grain yield/plant. In addition to, negative and highly significant correlation coefficient was recorded between this trait and grain yield per plant under level S1, S2 conditions. Abd-Allah (2000) illustrated that sterility $\%$ was negatively associated with grain yield/plant under both conditions.

\section{REFERENCES}

Abd Allah, A.A. (2000). Breeding studies on rice (Oryza sativa L.). Ph.D. Thesis, Fac. of Agric. Minufiya Univ. Shibin El-Kom, Egypt.

Abd El-Lattef, A.S.; A.B. El-Abd; A.A. Madyan and W.M.H. El-Khouby (2008). Inheritance of earliness, grain yield and some grain quality traits in rice (Oryza sativa L.). Under water deficiency conditions. J. Agric. Res. Kafr El-Sheikh Univ. 34(4): 83-105.

Bagheri, A. H.; G. A. Nematzadeh; S. A. Peighambari and M. Noroozi (2005). A study of combining ability and gene effect in rice cultivars through line/tester analysis. Iranian Journal of Agricultural Sciences, 36(4): 947953.

Chakraborty, R.; S. Chakraborty; B. K. Dutta and S. B. Paul (2010). Combining ability analysis for yield and yield components in bold grained rice (Oryza sativa L.) of Assam. Acta. Agron. (Palmira). 58(1): 9-13.

Dhakar, J. M. and V. Vinit. (2006). Combining ability analysis in rice (Oryza sativa L.). Crop Research Hisar, 31(3): 378-379. 
Dhillon, B.S. (1975). The application of partial diallel crosses in plant breeding-A review. Crop Improve., 2: 1-7.

El Abd, A. B., A. A. Abd Allah and A. A. El-Hissewy (2003). Studies on combining ability and heterosis for some physiological characters in rice (Oryza sativa L.). Proc. 10 th National Conference of Agronomy, Fac. of Environ. Sci., El-Arish, Suez Canal Univ., Egypt, 7-10, 81-93.

El Abd, A. B.; A. A. Abd Allah; S. M. Shehata; A. S. M. Abd El-Lateef and B. A. Zayed (2007). Heterosis and combining ability for yield and its components and some root characters in rice under water stress conditions. Proc. Fifth Plant Breeding Conference, May 27. Egypt. J. Plant Breeding, Special Issu,11 (2): 593-609.

El-Naem, S, M, A.(2010) . Breeding studies on rice (Oryza sativa L.) under water stress conditions. M.Sc. Agron. Fac. of Agric, Mansoura Univ., Egypt.

Falconer, D. S. and F. C. Mackey (1996). Introduction to Quantitative Genetics. Fourth Edition. Longman. New York.

Flowers, T. J. and A. R. Yeo (1981). Variability in the resistance of sodium chloride salinity within rice varieties. New Phytol., $88: 363-373$.

Ganapathy, S.; S. K. Ganesh; P. Vivekanandan; P. Shanmugasundaram and R. C. Babu (2007).Variability and interrelation ship between yield and physio - morphological traits in rice (Oryza sativa, L .) under moisture stress condition . Crop Research Hisar, 34 (1/3): 260-262.

Hammoud, S.A.A.M. (2004). Inheritance of some quantitative characters in rice (Oryza sativa L.). Ph. D. Thesis, Fac. Agric. Minufiya Univ., Shibin El-Kom, Egypt.

Hariprasanna, K.; F. U. Zaman ; A. K. Singh and S. M. S. Tomar (2006). Analysis of combining ability status among parents and hybrids in rice (Oryza sativa L.). Indian Journal of Genetics and Plant Breeding, 66(1): 28-30.

IRRI (1996). International Rice Research Descriptors for Rice. Los Banos, Laguna, Philippines. 52p.

Ismail, A. M.; M. J. Thomson; R. K. Singh; G. B. Gregorio and D. J. Mackill (2008). Designing rice varieties adapted to coastal areas of South and Southeast Asia. J Indian Soc. Coastal Agric. Res. 26: 69-73.

Jodon, N. E. (1938). Experiments on artificial hybridization of rice. J. Amer. Soc. Agron., 30: 249 - 305.

Kempthorne, O. (1957). An introduction to genetic statistics. John Wiley \& Sons.

Lutts, S.; J. M. Kinet and J. Bouharmont (1995). Changes in plant response to $\mathrm{NaCl}$ during development of rice (Oryza sativus L.) varieties differing in salinity resistance. J. Exp. Bot. 46, 1843-1852.

Manickavelu, A.; R. P. Gnanamalar; N. Nadarajan and S. K. Ganesh( 2006 ). Genetic variability studies on different genetic populations of rice under drought condition. Journal of Plant Sci. 1(4): 332-339.

Muthuramu, S.; S. Jebaraj; R. Ushakumari and M. Gnanasekaran (2010). Estimation of combining ability and heterosis for drought tolerance in different locations in rice (Oryza sativa L.). Electronic Journal of Plant Breeding, (1) 5: 1279-1285. 
Sultan, M. S. et al.

Negm, M.E.A.A (2011). Genetical studies on some physiological characters of salinity tolerance in rice. M.Sc. Thesis, Fac. of Agric., Kafr EISheikh University, Egypt.

Pradhan, S. K.; L.K. Boss and J. Meher (2006). Studies on gene action and combining ability analysis in Basmati rice. Journal of Centeral European Agriculture, 7 (2): 267-272.

Sharma, M. K., A. K. Sharma, R. K. Agrawal and A. K. Richharia. (2007). Combining ability and gene action for yield and quality traits in Ahu rices of Assam. Indian Journal of Genetics and Plant Breeding, 67 (3) 278-280.

Saxena, R. R.; R. R. Saxena; N. K. Motiramani; S. S. Nichal and R. K. Sahu. (2005). Studies on variability, heritability and genetic advance in scented rice germplasm accessions. Journal of Interacademicia 9(4): 487-489.

Sedeek, S. E. M. (2006). Breeding studies on rice. Ph.D. Thesis, Faculty of Agriculture, Kafr El-Sheikh, Tanta University, Egypt.

Shehata, S. M. (2004). Lines $x$ testers analysis of combining ability under salinity and drought conditions in rice. (Oryza sativa). Egyptian J. of Agric. Res., 82(1): 119-138.

Soroush, H. R. and A. Moumeni (2006). Genetic dissection of some important agronomic characters in rice using line $x$ tester analysis. Journal of Science and Technology of Agriculture and Natural Resources, 10(1): 177-187.

Verma, O. P. and H. K. Srivastava. (2004). Genetic component and combining ability analyses in relation to heterosis for yield and associated traits using three diverse rice-growing ecosystems. Field Crops Research, 88(2/3): 91-102.

Viswanathan Satheesh and K. Thiyagarajan. (2008). Combining ability studies for yield and yield components in rice (Oryza sativa L.) International J. of Plant Sci. (Muzaffarnagar),(31): 61-68.

Zeng, L.M M. C. Shannon and C. M. Grieve. (2002). Evaluation of salt tolerance in rice genotypes by multiple agronomic parameters. Euphytica, 127: 235-245. 


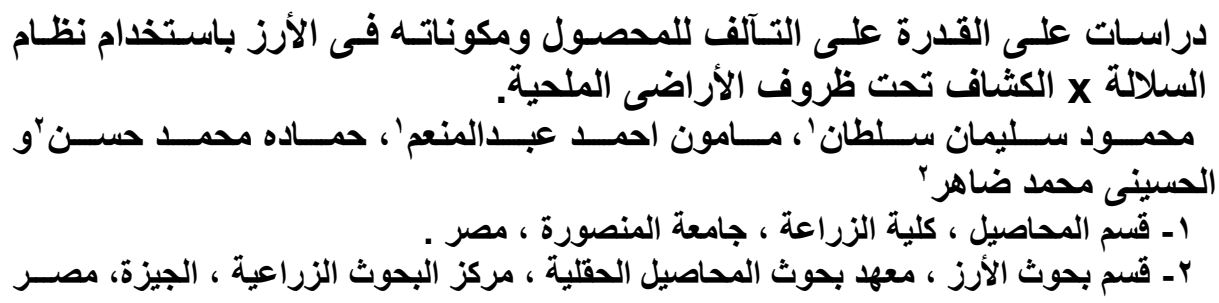

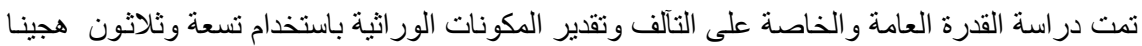

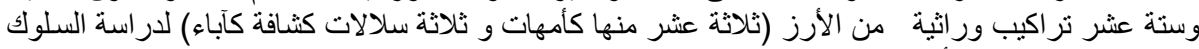

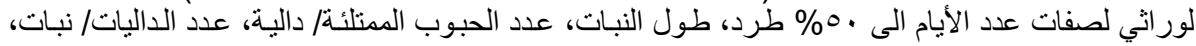

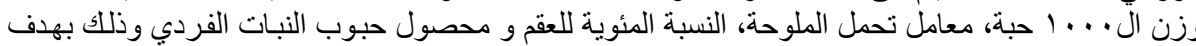

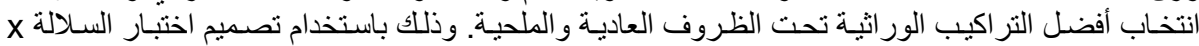

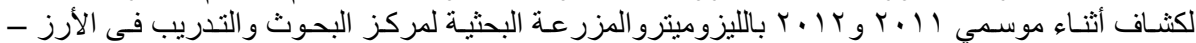

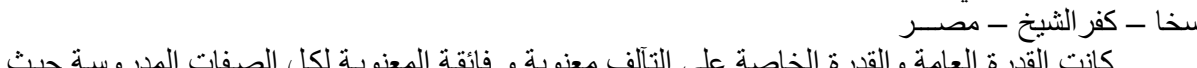

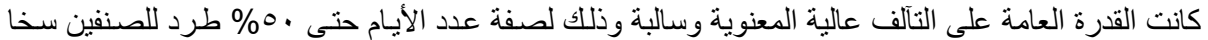

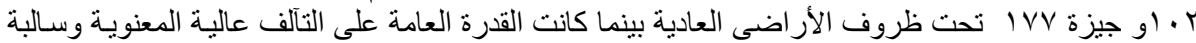

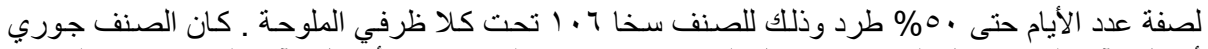

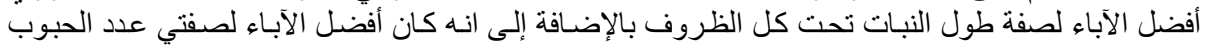

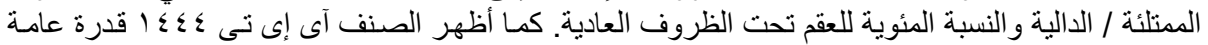

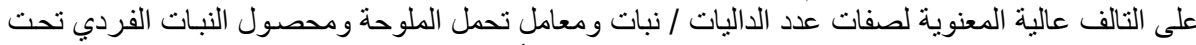

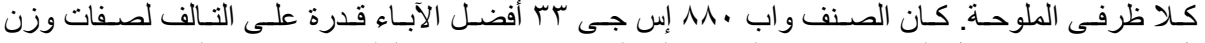

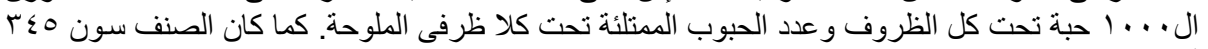

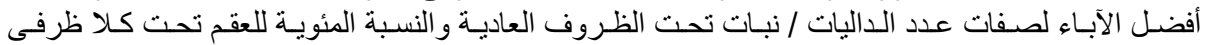

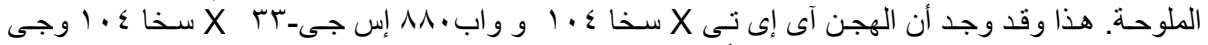

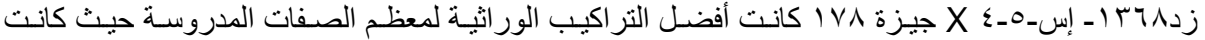

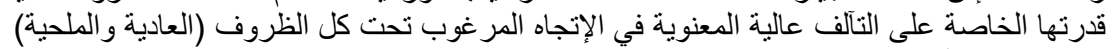

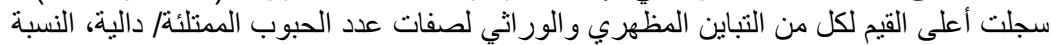

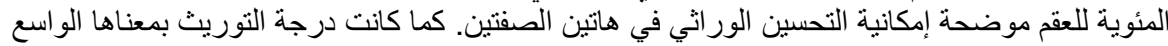

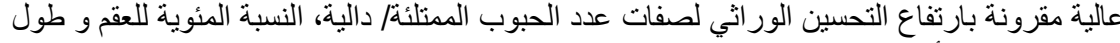

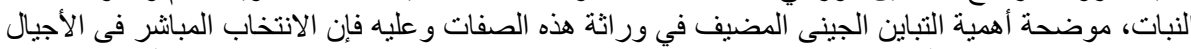

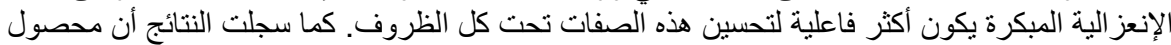

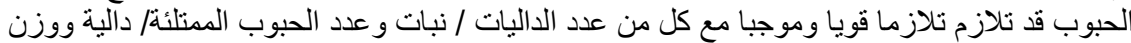

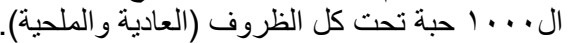

كلية الزراعة - جامعة المنصورة

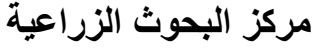

قام بتحكيم البحث

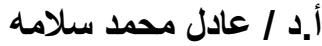

أ.د / أل العبد المعطى بسيونى العبد 